\title{
HIGHER DIVIDED SQUARES IN SECOND-QUADRANT SPECTRAL SEQUENCES ${ }^{1}$
}

BY

W. G. DWYER

\begin{abstract}
The geometric action of the Steenrod algebra on many mod 2 cohomology spectral sequences is complemented by the action of a completely different algebra.
\end{abstract}

1. Introduction. The purpose of this paper is to show that many second-quadrant mod 2 cohomology spectral sequences have a so-far unsuspected type of structurethe action of an algebra of "higher divided squares". This action is related in an essential way to the known action of the Steenrod algebra.

In more detail, suppose that $X$ is a cosimplicial space [2, p. 267], that is, a collection $\left\{X^{n}\right\}_{n>0}$ of spaces together with maps between them that satisfy the dual of the usual simplicial identities. There is a natural mod 2 second-quadrant cohomology spectral sequence $E_{r}^{-p, q}(p, q \geqslant 0)$ associated to $X$ which sometimes converges to an identifiable limit. The Eilenberg-Moore spectral sequence [8], the generalized Eilenberg-Moore spectral sequence [1], and a few other more exotic spectral sequences can be obtained in this way. Rector [8] and Smith [11] have shown that this spectral sequence admits Steenrod squaring operations $\mathrm{Sq}^{i}: E_{r}^{-p, q}$ $\rightarrow E_{r}^{-p, q+i}$ which can be nonzero, at $E_{2}$, for $i<q-1(p>0)$. This paper constructs higher divided square operations

$$
\delta_{i}: E_{2}^{-p, q} \rightarrow E_{2}^{-p-i, 2 q}, \quad 2<i<p,
$$

with the property that if the class $x$ in $E_{2}^{-p, q}$ survives to $E_{i+1}^{-p, q}$ then $d^{i}\left(\delta_{i} x\right)=$ $\mathrm{Sq}^{q-i+1}(x)$. In this way the higher divided square operations ensure that at $E_{\infty}$ the action of the Steenrod algebra is unstable with respect to total degree (which is $q-p)$.

The $\delta_{i}$ 's are constructed with the techniques of [4] and satisfy the Cartan formula and Adem relations derived there.

RelATIONSHIP TO EARLIER WORK. From a formal or technical point of view this paper develops for second-quadrant spectral sequences the analogue of Singer's theory of Steenrod squares in first-quadrant spectral sequences [9], [10]. Nevertheless the conclusions of the two theories are opposite to one another. There are too few "vertical" squaring operations in first-quadrant spectral sequences, and Singer constructs "horizontal" squaring operations that make up the deficit. There are too many vertical squaring operations in second-quadrant spectral sequences, and this

Received by the editors January 11, 1979 and, in revised form, September 27, 1979.

AMS (MOS) subject classifications (1970). Primary 55H05, 55H20, 18G40; Secondary 18 G30.

${ }^{1}$ This work was partially supported by National Science Foundation Grant MCS78-02977. 
paper constructs new horizontal operations that conspire with differentials to annihilate the extra vertical classes.

Let $\Delta$ be the category of the finite ordered sets $\{0,1, \ldots, n\}(n>0)$ and nondecreasing maps [6, p. 4] and let $\Delta^{\text {op }}$ be the opposite category. A mixed simplicial coalgebra is a functor from $\Delta^{\mathrm{op}} \times \Delta$ to the category of commutative $\mathrm{Z} / 2$-coalgebras. The singular complex of a cosimplicial space gives rise to a mixed simplicial coalgebra in a standard way [10, p. 352]. In order to emphasize the analogy with Singer's constructions most of the work in this paper will be done in the category of mixed simplicial coalgebras; in particular, this involves developing a purely algebraic approach to the geometric theory of Rector and Smith.

I would like to thank the referee for some very helpful comments.

2. Definitions and results. The constituents of a mixed simplicial object $X$ will be written $X_{q}^{p}$; the upper integer is the cosimplicial index and the lower the simplicial one. In the same vein, $d^{i}, s^{i}$ will stand for the "horizontal" coface and codegeneracy operators and $d_{i}, s_{i}$ for the "vertical" face and degeneracy operators. An augmentation for a mixed simplicial object $X$ is a simplicial object $R$ together with a simplicial map $\lambda: R \rightarrow X_{*}^{0}$ such that $d^{0} \lambda=d^{1} \lambda: R \rightarrow X_{*}^{1}$.

Any simplicial $\mathbf{Z} / 2$ module $R$ has an associated chain complex $C R$ given by $(C R)_{n}=R_{n}, d=\sum_{i=0}^{n} d_{i}$. Along the same lines, a mixed simplicial Z/2-module $X$ has an associated double chain complex $C X$ given by $(C X)_{-p, q}=X_{q}^{p}$, with horizontal differential $d^{h}=\sum_{i=0}^{p} d^{i}$ and vertical differential $d^{v}=\sum_{i=0}^{q} d_{i}$. It is sometimes useful to regard $C X$ (with a single subscript) as a singly indexed chain complex with a decreasing filtration

$$
F^{p}(C X)_{n}=\sum_{i>p}(C X)_{-i, n+i}, \quad d=d^{h}+d^{v}
$$

Note that from this point of view $(C X)_{*}$ is not positively graded.

Suppose that $X$ is a mixed simplicial $\mathbf{Z} / 2$-module. There is an increasingly filtered cochain complex $\operatorname{Hom}(C X, \mathbf{Z} / 2)$ defined by

$$
F_{p} \operatorname{Hom}^{n}(C X, \mathbf{Z} / 2)=\left[f:(C X)_{n} \rightarrow \mathbf{Z} / 2 \mid f\left(F^{p+1} C X\right)=0\right] .
$$

The spectral sequence $\left(E_{r}, d_{r}\right)$ of $X$ is by definition the spectral sequence of this filtered cochain complex (see $\S 5$ and $\left[5\right.$, p. 326]). Note that $\left(E_{r}, d_{r}\right)$ is a secondquadrant cohomology spectral sequence with $E_{0}^{-p, q}=\operatorname{Hom}\left(X_{q}^{p}, \mathrm{Z} / 2\right)$. If $X$ has an augmentation $\lambda: R \rightarrow X$ then $\lambda$ defines, in an obvious way, a $Z / 2$-homomorphism

$$
\lambda^{*}: H^{*} \operatorname{Hom}(C X, \mathrm{Z} / 2) \rightarrow H^{*} \operatorname{Hom}(C R, \mathbf{Z} / 2) \text {. }
$$

When $\lambda^{*}$ is an isomorphism the cohomology $H^{*} \operatorname{Hom}(C R, \mathbf{Z} / 2)$ is filtered and is the "target" of the spectral sequence $\left(E_{r}, d_{r}\right)$. The spectral sequence need not converge, but in any case let

$$
\rho: F_{p} H^{q-p} \operatorname{Hom}(C R, \mathrm{Z} / 2) \rightarrow E_{\infty}^{-p, q}
$$

be the natural projection.

Suppose now that $X$ is a mixed simplicial coalgebra and that $R$ is a simplicial coalgebra (all coalgebras are commutative coalgebras over $Z / 2$ as in [9]). $\$ 4$ 
contains a construction of products and Steenrod operations both on $H^{*} \operatorname{Hom}(C R, \mathbf{Z} / 2)$ and on $H^{*} \operatorname{Hom}(C X, \mathrm{Z} / 2)$.

2.1. Proposition. If $\lambda: R \rightarrow X$ is an augmentation, then $\lambda^{*}$ preserves products and Steenrod operations.

$\$ 5$ contains a construction of products

$$
E_{r}^{-p, q} \otimes E_{r}^{-s, t} \rightarrow E_{r}^{-(p+s), q+t} \quad(r \geqslant 2)
$$

in the spectral sequence $\left(E_{r}, d_{r}\right)$ of $X$, as well as a construction of Steenrod operations

$$
\mathrm{Sq}^{k}: E_{r}^{-p, q} \rightarrow E_{r}^{-p, q+k} \quad(r \geqslant 2)
$$

2.2. Proposition. Products and Steenrod operations on $E_{2}$ determine products and Steenrod operations on $E_{r}$ for all $r \geqslant 2$. For example, if $u \in E_{2}$ survives to $E_{r}$ and represents $[u] \in E_{r}$, then $\mathrm{Sq}^{k} u$ survives to $E_{r}$ and $\left[\mathrm{Sq}^{k} u\right]=\mathrm{Sq}^{k}[u]$.

2.3. Proposition. Under the above product pairing $\left(E_{r}, d_{r}\right)$ is a spectral sequence of differential algebras. The Steenrod squares commute with the differentials.

It follows that there are products and squaring operations on $E_{\infty}$.

2.4. Proposition. Suppose that $\lambda: R \rightarrow X$ is an augmentation and that $\lambda^{*}$ is an isomorphism. Suppose that $u \in F_{p} H^{q-p} \operatorname{Hom}(C R, \mathrm{Z} / 2)$ and $v \in$ $F_{r} H^{s-r} \operatorname{Hom}(C R, \mathrm{Z} / 2)$. Then $u v \in F_{p+r} H^{*} \operatorname{Hom}(C R, \mathrm{Z} / 2)$ and $\rho(u v)=\rho(u) \rho(v)$. Moreover $\mathrm{Sq}^{k} u \in F_{p} H^{*} \operatorname{Hom}(C R, \mathrm{Z} / 2)$ and $\rho \mathrm{Sq}^{k} u=\mathrm{Sq}^{k} \rho(u)$.

Fix $r \geqslant 2$ and pick $s \geqslant r$. Let $B_{s}^{-p, q}$ denote the subgroup of $E_{r}^{-p, q}$ containing those elements $x$ which survive to $E_{s}^{-p, q}$ and have zero residue class $[x]$ in $E_{s}^{-p, q}$. By definition, an operation $\delta: E_{r}^{-p, q} \rightarrow E_{r}^{-a, b}$ of indeterminacy $s$ is a map $E_{r}^{-p, q} \rightarrow$ $E_{r}^{-a, b} / B_{s}^{-a, b}$.

$\$ 5$ contains a definition of higher divided square operations

$$
\delta_{i}: E_{r}^{-p, q} \rightarrow E_{r}^{-p-i, 2 q}, \quad 2<r<i<p,
$$

of indeterminacy $2 r-2$. Note, in particular, that at $E_{2}$ the indeterminacy is trivial.

2.5. Proposition. Higher divided square operations on $E_{2}$ determine higher divided square operations on $E_{r}$ for $r>2$. In other words, if $u \in E_{2}^{-p, q}$ survives to $E_{r}$ and represents $[u] \in E_{r}^{-p, q}$, then for $r \leqslant i \leqslant p$ the class $\delta_{i} u$ survives to $E_{r}$ and $\delta_{i}[u]=$ $\left[\delta_{i} u\right]$ modulo the appropriate indeterminacy.

REMARK. The indeterminacy of the operation $\delta_{i}$ always lies in the kernel of all spectral sequence differentials.

To avoid complicated notation, in the next statement no distinction is made between an element $u$ in $E_{r}^{-p, q}$ that survives to $E_{s}$ and its residue class in $E_{s}^{-p, q}$. 
2.6. Proposition. Suppose that $u \in E_{r}^{-p, q}(r<p)$ and that $d_{r} u=v$, with $v \in$ $E_{r}^{-p+r, q-r+1}$. Then

(a) for $r \leqslant i \leqslant \min \{2 r-2, p-1\}$, the classes $\delta_{i} u$ and $\mathrm{Sq}^{q-i+1} u$ both survive to $E_{i}$ and $d_{i} \delta_{i} u=\mathrm{Sq}^{q-i+1} u$,

(b) if $p \geqslant 2 r$, so that $\delta_{r} v$ is defined, the classes $\delta_{2 r-1}(u)$ and $\mathrm{Sq}^{q-2 r+2} u+\delta_{r} v$ both survive to $E_{2 r-1}$ and

$$
d_{2 r-1} \delta_{2 r-1}(u)=\mathrm{Sq}^{q-2 r+2} u+\delta_{r} v \text {, }
$$

(c) for $2 r \leqslant i<p$ the classes $\delta_{i} u$ and $\delta_{i-r+1}(v)$ both survive to $E_{2 r-1}$ and $d_{2 r-1} \delta_{i} u=\delta_{i-r+1}(v)$,

(d) if $r<p$ then $d_{r} \delta_{p} u=u v$ while if $r=p$ then $d_{r} \delta_{p} u=u v+\mathrm{Sq}^{q-p+1} u$.

REMARK. If $u \in E_{r}$ then at $E_{2 r-1}$ all of the indeterminacy in the value of $\delta_{i} u$ has disappeared.

2.7. Proposition. The action of the Steenrod operations on $E_{2}$ satisfies the Cartan formula and the Adem relations (both, in general, interpreted with $\mathrm{Sq}^{0} \neq 1$ ). The action of the higher divided squares on $E_{2}$ satisfies the relations of $[4,2.1]$. The two actions satisfy the commutation relation $\mathrm{Sq}^{k} \delta_{i}=\delta_{i} \mathrm{Sq}^{k / 2}$ where $\mathrm{Sq}^{k / 2}=0$ for $k$ odd.

The relations of $[4,2.1]$ imply that if $u \in E_{2}^{-p, q}$ and $v \in E_{2}^{-r, s}$ the following three properties hold.

(i) If $p=r$ and $q=s$, then

$$
\delta_{i}(u+v)=\delta_{i} u+\delta_{i} v, \quad \delta_{p}(u+v)=\delta_{p} u+\delta_{p} v+u v, \quad 2<i<p .
$$

(ii) If $2 \leqslant i \leqslant p+r$, then

$$
\delta_{i}(u v)= \begin{cases}v^{2} \delta_{i}(u) & \text { if } r=0 \\ u^{2} \delta_{i}(v) & \text { if } p=0 \\ 0 & \text { otherwise }\end{cases}
$$

(iii) If $2 \leqslant i \leqslant p$ and $2 \leqslant j \leqslant 2 i$, then

$$
\delta_{j} \delta_{i}(u)=\sum_{\frac{j+1}{2}<k<i-1}\left(\begin{array}{c}
i-j+k-1 \\
i-k
\end{array}\right) \delta_{i+j-k} \delta_{k}(u) .
$$

3. Chain and cochain diagonal approximations. The aim of this section is to recall the properties of some natural chain and cochain homomorphisms.

If $R$ and $S$ are simplicial $\mathrm{Z} / 2$-modules, let $R \otimes S$ denote the dimensionwise tensor product $(R \otimes S)_{n}=R_{n} \otimes S_{n}$ with the tensor product face and degeneracy operators. If $A$ and $B$ are graded $\mathrm{Z} / 2$-modules, let $A \hat{\otimes} B$ denote the graded tensor product

$$
(A \hat{\otimes} B)_{n}=\sum_{i+j=n}\left(A_{i} \otimes B_{j}\right)
$$

The Leibnitz differential

$$
d(a \otimes b)=d(a) \otimes b+a \otimes d(b)
$$

makes $A \hat{\otimes} B$ into a chain complex if $A$ and $B$ are chain complexes. 
Let $T$ denote the switching map

$$
C(R \otimes S) \rightarrow C(S \otimes R) \text { or } C R \hat{\otimes} C S \rightarrow C S \hat{\otimes} C R .
$$

\subsection{LEMMA. There exist natural maps}

$$
D_{k}: C(R \otimes S)_{i} \rightarrow(C R \hat{\otimes} C S)_{i+k}, \quad i, k \geqslant 0,
$$

such that

(i) $D_{0}$ is a chain homotopy equivalence which induces the identity map $C(R \otimes S)_{0}$ $\rightarrow(C R \hat{\otimes} C S)_{0}$, and

(ii) $d D_{k}+D_{k} d=D_{k-1}+T D_{k-1} T(k>0)$.

The collection $\left\{D_{k}\right\}$ is a simplicial Eilenberg-Zilber map in the sense of [9] and is constructed in [3]. It is convenient to adopt the convention that $D_{k}=0$ for $k<0$.

Suppose now that $U=\left\{U^{i}\right\}$ is a cosimplicial Z/2-module [2, p. 267]. Associated to $U$ is a cochain complex $C U$ given by $(C U)^{n}=U^{n}, d=\sum_{i=0}^{n+1} d^{i}$. If $V$ is another cosimplicial Z/2-module, the "codimensionwise" tensor product $U \otimes V$ and the graded tensor product $C U \hat{\otimes} C V$ are defined in the obvious way.

Let $T$ be one of the switching maps. $C(U \otimes V) \rightarrow C(V \otimes U)$ or $C U \hat{\otimes} C V \rightarrow$ $C V \hat{\otimes} C U$. A map $\phi^{k}: C(U \otimes V) \rightarrow C U \hat{\otimes} C V$ of degree $k$ is said to be admissible if

(a) $\phi^{k} T=T \phi^{k}$

(b) the restriction of $\phi^{k}$ to $U^{i} \otimes V^{i}$ is zero unless $i=k$,

(c) the image of $\phi^{k}: U^{k} \otimes V^{k} \rightarrow(C U \hat{\otimes} C V)^{2 k}$ is contained in $(C U)^{k} \otimes(C V)^{k}$ $=U^{k} \otimes V^{k}$, and

(d) the map $U^{k} \otimes V^{k} \rightarrow U^{k} \otimes V^{k}$ induced by $\phi^{k}$ according to (c) restricts to the identity on the intersection of the kernels of the codegeneracy maps

$$
s^{k}: U^{k} \otimes V^{k} \rightarrow U^{k-1} \otimes V^{k-1}, \quad 0<i<k-1 .
$$

\subsection{LEMMA. There exist natural maps}

$$
\Delta^{k}: C(U \otimes V)^{i} \rightarrow(C U \hat{\otimes} C V)^{i+k}, \quad 0<k<i,
$$

and natural admissible maps $\phi^{k}$ of degree $k(k>0)$ such that

(i) the map $\Delta=\Delta^{0}+T \Delta^{0} T+\phi^{0}$ is a cochain homotopy equivalence that induces the identity map $C(U \otimes V)^{0} \rightarrow(C U \hat{\otimes} C V)^{0}$, and

(ii) $\Delta^{k-1} d+d \Delta^{k-1}=\Delta^{k}+T \Delta^{k} T+\phi^{k}(k>0)$.

REMARKS. Equation (ii) only makes sense when applied to an element $x$ of degree $>k$, since $\Delta^{k}(x)$ is not defined otherwise. The awkward definition of admissible map could be avoided by working systematically with normalized cochain complexes, but this would lead to other complications.

Proof of Lemma 3.2. Let $R$ and $S$ be the simplicial $\mathrm{Z} / 2$-modules which are the $\mathrm{Z} / 2$-duals of $U$ and $V$, and let $\mathrm{N}(R \otimes S)$ be the normalized chain complex of the simplicial $\mathrm{Z} / 2$-module $R \otimes S[5$, p. 236]. The natural projection $C(R \otimes S) \rightarrow$ $\mathbf{N}(R \otimes S)$ has a natural section [6, p. 94], so that if $U$ and $V$ are finitely generated in each codimension the maps of Lemma 3.2 can be constructed by composing the maps $C R \hat{\otimes} C S \rightarrow \mathrm{N}(R \otimes S)$ of [4, Proposition 3.3] with this section and dualizing. The general case is handled by using the fact that $U$ and $V$ can each be 
expressed as a direct limit of cosimplicial Z/2-modules which are finitely generated in each codimension.

4. Products and Steenrod operations. This section gives the construction of products and Steenrod operations, first for simplicial and then for mixed simplicial coalgebras.

Suppose that $R$ is a simplicial coalgebra, and let

$$
\chi: \operatorname{Hom}(C R, \mathbf{Z} / 2) \hat{\otimes} \operatorname{Hom}(C R, \mathbf{Z} / 2) \rightarrow \operatorname{Hom}(C R \hat{\otimes} C R, \mathbf{Z} / 2)
$$

be the natural pairing map. Let $\psi: R \rightarrow R \otimes R$ be the coproduct, and define cochain operations

$$
\begin{gathered}
\mu: \operatorname{Hom}^{p}(C R, \mathbf{Z} / 2) \otimes \operatorname{Hom}^{q}(C R, \mathbf{Z} / 2) \rightarrow \operatorname{Hom}^{p+q}(C R, \mathbf{Z} / 2), \\
S^{k}: \operatorname{Hom}^{n}(C R, \mathbf{Z} / 2) \rightarrow \operatorname{Hom}^{n+k}(C R, \mathbf{Z} / 2)
\end{gathered}
$$

by the formulas $\mu(x \otimes y)=\psi^{*} D_{0}^{*} \chi(x \otimes y)$,

$$
S^{k}(x)=\psi^{*} D_{n-k}^{*} \chi(x \otimes x)+\psi^{*} D_{n-k+1}^{*} \chi(x \otimes d x) .
$$

Then $\mu$ and $S^{k}$ pass to products and Steenrod squares on $H^{*} \operatorname{Hom}(C R, Z / 2)$. These have all of the usual properties, except that in general $\mathrm{Sq}^{0} \neq 1$ [7, p. 198].

The mixed simplicial case needs a few preliminaries. Suppose first that $X$ and $Y$ are mixed simplicial $Z / 2$-modules. The componentwise tensor product of $X$ and $Y$ is written $X \otimes Y$ : it is a mixed simplicial $\mathbf{Z} / 2$-module with the usual tensor product face, coface, - etc. operators. The bigraded tensor product of the double complexes $C X$ and $C Y$ is written $C X \hat{\otimes} C Y$. It is the double complex defined by

$$
(C X \hat{\otimes} C Y)_{-p, q}=\sum_{\substack{i+j=p \\ k+l=q}}(C X)_{-i, k} \otimes(C Y)_{-j, l}
$$

and has vertical and horizontal differentials which are given by the Leibnitz rule. There is an intermediate object, called the horizontal tensor product of $X$ and $Y$. This is the double complex $C\left(X \otimes_{H} Y\right)$ given by

$$
C\left(X \otimes_{H} Y\right)_{-p, q}=\sum_{i+j=p} X_{q}^{i} \otimes Y_{q}^{j}
$$

with horizontal differential $\left(\sum d^{i}\right) \otimes 1+1 \otimes\left(\Sigma d^{i}\right)$ and vertical differential $\Sigma\left(d_{i} \otimes d_{i}\right)$.

Define homomorphisms

$$
H: C(X \otimes Y) \rightarrow C\left(X \otimes_{H} Y\right), \quad G_{s}: C\left(X \otimes_{H} Y\right) \rightarrow C S \hat{\otimes} C Y,
$$

where $H$ is homogeneous of bidegree $(0,0)$ and $G_{s}$ is homogeneous of bidegree $(0, s)$, as follows. The restriction of $H$ to $C(X \otimes Y)_{-p, q}$ is

$$
\Delta\left(X_{1}^{*}, Y_{q}^{*}\right): X_{q}^{p} \otimes Y_{q}^{p} \rightarrow \sum_{i+j=p} X_{q}^{i} \otimes Y_{q}^{j}
$$

and the restriction of $G_{s}$ to the summand $X_{q}^{i} \otimes Y_{q}^{j}$ of $C\left(X \otimes_{H} Y\right)_{-p, q}(i+j=p)$ is

$$
D_{s}\left(X_{*}^{i}, X_{*}^{j}\right): X_{q}^{i} \otimes Y_{q}^{j} \rightarrow \sum_{k+l=q+s} X_{k}^{i} \otimes Y_{l}^{j}
$$


Let $T$ denote the appropriate switching map. It follows that

$$
\begin{array}{cl}
d^{v} G_{s}+G_{s} d^{v}=G_{s-1}+T G_{s-1} T, & d^{h} G_{s}=G_{s} d^{h}, \\
d^{v} H=H d^{v}, \quad d^{h} H=H d^{h}, & H=T H T .
\end{array}
$$

Define maps $K_{k}: C(X \otimes Y) \rightarrow C X \hat{\otimes} C Y$ homogeneous of bidegree $(0, k)$ by $K_{k}=G_{k} H$. A computation shows that $d K_{k}+K_{k} d=K_{k-1}+T K_{k-1} T$, where $d=$ $d^{h}+d^{v}$ is the total differential.

Suppose that $X$ is a mixed simplicial coalgebra, and let

$$
\chi: \operatorname{Hom}(C X, \mathbf{z} / 2) \hat{\otimes} \operatorname{Hom}(C X, \mathbf{z} / 2) \rightarrow \operatorname{Hom}(C X \hat{\otimes} C X, \mathbf{z} / 2)
$$

be the natural pairing map. Let $\psi: X \rightarrow X \otimes X$ be the coproduct, and define cochain operations

$$
\begin{gathered}
\mu: \operatorname{Hom}^{p}(C X, \mathbf{Z} / 2) \otimes \operatorname{Hom}^{q}(C X, \mathbf{Z} / 2) \rightarrow \operatorname{Hom}^{p+q}(C X, \mathbf{Z} / 2), \\
S^{k}: \operatorname{Hom}^{n}(C X, \mathbf{Z} / 2) \rightarrow \operatorname{Hom}^{n+k}(C X, \mathbf{Z} / 2)
\end{gathered}
$$

by the formulas

$$
\mu(x \otimes y)=\psi^{*} K_{0}^{*} \chi(x \otimes y), \quad S^{k}(x)=\psi^{*} K_{n-k}^{*} \chi(x \otimes x)+\psi^{*} K_{n-k+1}^{*} \chi(x \otimes d x) .
$$

Then $\mu$ and $S^{k}$ pass to products and Steenrod squares on $H^{*} \operatorname{Hom}(C X, Z / 2)$.

Proposition 2.1 can be proved in the same way as [9, Proposition 1.1]. The main ingredient is (i) of Lemma 3.2.

Note that the squaring operations on $H^{*} \operatorname{Hom}(C X, \mathrm{Z} / 2)$ satisfy the "instability" condition $\mathrm{Sq}^{k} x=0$ if $k>\operatorname{dim} x$, since $K_{k}^{*}=0$ if $k<0$.

5. The spectral sequence of a mixed simplicial coalgebra. Suppose that $X$ is a mixed simplicial coalgebra. The spectral sequence $\left\{E_{r}^{-p, q}, d_{r}\right\}$ of $X$ is defined in the usual way.

$$
E_{r}^{-p, q}=Z_{r}^{-p, q} /\left[d Z_{r-1}^{-p-r+1, q+r-2}+Z_{r-1}^{-p+1, q-1}\right]
$$

where

$$
Z_{r}^{-p, q}=\left\{x \in F_{p} \operatorname{Hom}^{q-p}(C X, \mathbf{Z} / 2) \mid d x \in F_{p-r} \operatorname{Hom}(C X, \mathbf{Z} / 2)\right\} .
$$

The differential $d$ on $\operatorname{Hom}(C X, Z / 2)$ induces

$$
d_{r}: E_{r}^{-p, q} \rightarrow E_{r}^{-p+r, q-r+1}
$$

Let $\operatorname{Hom}(C X, \mathbf{Z} / 2) \hat{\otimes} \operatorname{Hom}(C X, \mathbf{Z} / 2)$ have the standard increasing filtration associated with a tensor product of increasingly filtered complexes. Then it is clear that the cochain multiplication map

$$
\mu: \operatorname{Hom}(C X, \mathbf{Z} / 2) \hat{\otimes} \operatorname{Hom}(C X, \mathbf{Z} / 2) \rightarrow \operatorname{Hom}(C X, \mathbf{Z} / 2)
$$

is filtration-preserving, and so passes to a pairing on the spectral sequence $\left(E_{r}, d_{r}\right)$ of $X$. It follows easily that this pairing has the properties stated in Propositions 2.2, 2.3 and 2.4.

The cochain level Steenrod maps

$$
S^{k}: \operatorname{Hom}^{n}(C X, Z / 2) \rightarrow \operatorname{Hom}^{n+k}(C X, Z / 2)
$$

are filtration doubling, and so can be used to define spectral sequence operations $E_{r}^{-p, q} \rightarrow E_{r}^{-2 p p+q+k}$ of indeterminancy $2 r-1$. These operations are identically 
zero unless $p=0$. One way to overcome this difficulty and also eliminate the problem of indeterminancy is to deform the maps $S^{k}$ so that they preserve filtration. This leads directly to the construction of higher divided squares, and to spectral sequence Steenrod operations that are not unstable with respect to total degree.

For the moment, let $X$ and $Y$ be mixed simplicial $Z / 2$-modules as in \$4. Define homomorphisms

$$
P^{k}, H^{k}: C(X \otimes Y) \rightarrow C\left(X \otimes_{H} Y\right) \quad(k>0)
$$

which are homogeneous of degree $(-k, 0)$ as follows. The restriction of $P^{k}$ to $C(X \otimes Y)_{-p, q}$ is

$$
\phi^{k}\left(X_{q}^{*}, Y_{q}^{*}\right): X_{q}^{p} \otimes Y_{q}^{p} \rightarrow \sum_{i+j=p+k} X_{q}^{i} \otimes Y_{q}^{j}
$$

and the restriction of $H^{k}$ to $C(X \otimes Y)_{-p, q}$ is zero if $p<k$ and is otherwise equal to

$$
\Delta^{k}\left(X_{q}^{*}, Y_{q}^{*}\right): X_{q}^{p} \otimes Y_{q}^{p} \rightarrow \sum_{i+j=p+k} X_{q}^{i} \otimes Y_{q}^{j} .
$$

Give $C\left(X \otimes_{H} Y\right)$, considered as a singly indexed complex, the usual decreasing filtration

$$
F^{P} C\left(X \otimes_{H} Y\right)_{n}=\sum_{i>p} C\left(X \otimes_{H} Y\right)_{-i, n+i}
$$

and let $T$ denote any one of the appropriate switching maps. The following properties of the maps $P^{k}, H^{k}$ are either trivial or are immediate consequences of the formulas in $\$ 3$.

$$
\begin{gathered}
d^{v} P^{k}=P^{k} d^{0}, \quad P^{k} T P^{k}, \\
d^{v} H^{k}=H^{k} d^{v}, \quad H^{0}+T H^{0} T+P^{0}+H .
\end{gathered}
$$

In addition, it is easy to see that if $x \in F^{P} C(X \otimes Y)$ then modulo $F^{2 p+1} C\left(X \otimes_{H} Y\right)$ there are congruences

$$
\begin{aligned}
P^{k}(X) & \equiv 0 \quad(k \neq p), \\
d^{h} H^{k}(x)+H^{k} d^{h}(x) & \equiv H^{k+1}(x)+T H^{k+1} T(x)+P^{k+1}(x) .
\end{aligned}
$$

For any integer $k$ (positive or negative) define a map

$$
J_{k}: C(X \otimes Y) \rightarrow C X \hat{\otimes} C Y
$$

homogeneous of degree $k$ with respect to total degree, by

$$
J_{k}(x)=\sum_{\substack{i-j=k \\ i, j>0}} G_{i} T^{i} H^{j} T^{i}(x) .
$$

(Because of the way in which the $H^{j}$ were defined only a finite number of terms on the right-hand side are nonzero for any particular $x$.) Let $d=d^{h}+d^{0}$ be the total differential. A calculation shows that for $x \in F^{P} C(X \otimes Y)$ the congruence

$$
d J_{k}(x)+J_{k} d(x) \equiv J_{k-1}(x)+T J_{k-1} T(x)+G_{k-1} H(x)+G_{k+p-1} P^{P}(x)
$$

holds modulo $F^{2 p+1}(C X \hat{\otimes} C Y)$. 
Suppose that $X$ is a mixed simplicial coalgebra with diagonal map $\psi$ and pairing $\chi$ as in $\$ 4$. For any integer $k$ define a cochain operation

$$
\Sigma^{k}: \operatorname{Hom}^{n}(C X, \mathbf{Z} / 2) \rightarrow \operatorname{Hom}^{n+k}(C X, \mathbf{Z} / 2)
$$

by

$$
\Sigma^{k}(x)=\psi^{*} J_{n-k}^{*} \chi(x \otimes x)+\psi^{*} J_{n-k+1}^{*} \chi(x \otimes d x) .
$$

Dualizing the congruences above shows that for $x \in F_{p} \operatorname{Hom}^{n}(C X, Z / 2)$ the congruence

$$
\begin{aligned}
\Sigma^{k} d(x) & +d \Sigma^{k}(x) \\
& \equiv S^{k+1}(x)+\psi^{*}\left(P^{p}\right)^{*}\left(G_{n-k+p-1}^{*} \chi(x \otimes x)+G_{n-k+p}^{*} \chi(x \otimes d x)\right)
\end{aligned}
$$

holds modulo $F_{p-1} \operatorname{Hom}(C X, \mathrm{Z} / 2)$.

Let $\bar{S}^{k}: \operatorname{Hom}^{n}(C X, \mathbf{Z} / 2) \rightarrow \operatorname{Hom}^{n+k}(C X, \mathbf{Z} / 2)$ be the cochain map defined by

$$
\bar{S}^{k}(x)=S^{k}(x)+\Sigma^{k-1} d(x)+d \Sigma^{k-1}(x) .
$$

Then $\bar{S}^{k}$ is filtration-preserving, $d \bar{S}^{k}=\bar{S}^{k} d$, and, for any cocycle $x \in$ $\operatorname{Hom}(C X, \mathrm{Z} / 2), \bar{S}^{k}(x)$ is visibly cohomologous to $S^{k}(x)$. It follows immediately that the maps $\bar{S}^{k}$ pass to Steenrod operation $E_{r}^{-p, q} \rightarrow E_{r}^{-p, q+k}$ that have all of the properties described in Propositions 2.2-2.4. These operations may well be nonzero even if $k>q-p$.

Write $F_{j}$ for $F_{j} \operatorname{Hom}(C X, \mathrm{Z} / 2)$ and suppose that $x \in Z_{1}^{-p, q}$, so that $x \in F_{p}$ and $d x \in F_{p-1}$. A straightforward filtration calculation shows that

$$
\begin{aligned}
\Sigma^{k}(x) & \in F_{2 p}, \quad k<q-p, \\
& \in F_{p+\sigma-k}, \quad q-p<k \leqslant q, \\
& \in F_{p}, \quad q<k .
\end{aligned}
$$

In particular if $x \in Z_{r}^{-p, q}(r \geqslant 1)$, then for $2<k<p$ the element $\Sigma^{q-k}(x)$ lies in $F_{p+k}$. A little manipulation of the formulas above shows that for $2<k<p$ the congruences

$$
d \Sigma^{q-k}(x)+\Sigma^{q-k} d(x) \equiv \psi^{*}\left(P^{p}\right)^{*}\left(G_{k-1}^{*} \chi(x \otimes x)+G_{k}^{*} \chi(x \otimes d x)\right)
$$

holds modulo $F_{p-1}$. To see this, use the fact that the cochain operations $S^{k}$ satisfy $S^{k} x=0$ if $k>\operatorname{dim} x+1$. The same congruence holds for $k=p$ if the term $\psi^{*} H^{*} G_{0}^{*} \chi(x \otimes d x)$ is added to the right-hand side.

If $x \in Z_{r}^{-p, q}(r>2)$ represents $\{x\} \in E_{r}^{-p, q}$, define $\delta_{i}(\{x\})$ for $r<i<p$ to be the residue class in $E_{r}^{-p-i, 2 q}$ of the element $\Sigma^{q-i}(x) \in Z_{r}^{-p-i, 2 q}$. The indeterminacy properties of the operations $\delta_{i}$ and all of the properties listed in Propositions 2.5 and 2.6 are straightforward consequences of the congruences listed above. It is useful to keep in mind that at $E_{2}$ the operations $\delta_{i}$ are linear or at worst quadratic (Proposition 2.7, see §6).

REMARK. An operation " $\delta_{i}$ " exists on the $E_{1}$-level, in the sense that if $x \in Z_{1}^{-p, q}$ $(p>0)$ represents a class $\{x\} \in E_{1}^{-p, q}$ which survives to $E_{2}$, then $\Sigma^{q-1}(x)$ belongs to $Z^{-p-1,2 q}$ and $d_{1}\left\{\Sigma^{q-1}(x)\right\}=\operatorname{Sq}^{q}\{x\}$. 
6. Operations on the $E_{2}$-term. Suppose that $X=\left\{X_{q}^{p}\right\}$ is a mixed simplicial coalgebra. For each $p \geqslant 0,\left\{X_{*}^{p}\right\}$ is a simplicial coalgebra; let $A(p)$ denote the corresponding cohomology algebra $H^{*} \operatorname{Hom}\left(C\left(X_{*}^{p}\right), \mathbf{Z} / 2\right)$. The transposes of the coface and codegeneracy operators of $X$ give the collection $A=\{A(p)\}$ the structure of a simplicial object in the category of graded commutative $Z / 2$ algebras, and the $E_{2}$-term of the spectral sequence of $X$ can be computed in terms of the (graded) homotopy groups of $A$.

$$
E_{2}^{-p, q}=\left(\pi_{p} A\right)_{q}
$$

(The homotopy groups of a simplicial commutative $\mathbf{Z} / 2$-algebra are by definition the homology groups of the associated normalized chain complex [5, p. 236]. The homotopy groups of a graded algebra themselves inherit a natural grading.)

The machinery of [4], slightly refined to take the grading of $A$ into account, provides algebraically defined higher divided square operations

$$
\delta_{i}^{\text {alg: }}\left(\pi_{p} A\right)_{q} \rightarrow\left(\pi_{p+i} A\right)_{2 q}, \quad 2<i<p,
$$

and algebraically defined pairing maps

$$
\left(\pi_{p} A\right)_{q} \otimes\left(\pi_{s} A\right)_{t} \rightarrow\left(\pi_{p+s} A\right)_{q+t}
$$

In addition, the Steenrod operations (\$4)

$$
\mathrm{Sq}^{i}: A(p)_{q} \rightarrow A(p)_{q+i}
$$

commute with face and degeneracy operators and so pass to operations

$$
\mathrm{Sq}_{\mathrm{alg}}^{i}:\left(\pi_{p} A\right)_{q} \rightarrow\left(\pi_{p} A\right)_{q+i}
$$

6.2. LemMA. At $E_{2}$ the operations $\mathrm{Sq}^{i}$ and $\delta_{i}$ constructed in $\$ 5$ agree via (6.1) with the operations $\mathrm{Sq}_{\mathrm{alg}}^{i}$ and $\delta_{i}^{\mathrm{alg}}$. At $E_{2}$ the pairing constructed in $\$ 5$ agrees via (6.1) with the algebraic pairing described above.

The proof of this is essentially the same as the proof of [9, Proposition 5.1]. It involves inspecting the leading terms of the cochain formulas for $\operatorname{Sq}^{i}\{x\}, \delta_{i}\{x\}$ and $\{x\} \cdot\{y\}$ and using, in the $\mathrm{Sq}^{i}$ case, property (d) of an "admissible map" (§3).

Proof of Proposition 2.7. If $R$ is a simplicial coalgebra, the action of the Steenrod squares on $H^{*} \operatorname{Hom}(C R, \mathrm{Z} / 2)$ satisfies the Adem relations and the Cartan formula (both, in general, interpreted with $\mathrm{Sq}^{0} \neq 1$ ) [7, §7]. It follows from Lemma 6.2 that the action of the Steenrod squares on $E_{2}$ similarly satisfies the Adem relations. It also follows directly from Lemma 6.2 that the action of the higher divided squares on $E_{2}$ satisfies the relations of [4, Theorem 2.1]. For the remaining statements, let $\mathrm{Sq}=\mathrm{Sq}^{0}+\mathrm{Sq}^{1}+\mathrm{Sq}^{2}+\cdots$ and note that by the Cartan formula $\mathrm{Sq}$ acts as a simplicial algebra endomorphism of $A$. If $\mathrm{Sq}_{\#}$ denotes the induced map on $\pi_{*} A$ it follows by naturality that for $x \in \pi_{p} A, y \in \pi_{q} A$ there are equalities

$$
\mathrm{Sq}_{\#} \delta_{i}(x)=\delta_{i} \mathrm{Sq}_{\#}(x), \quad \mathrm{Sq}_{\#}(x y)=\mathrm{Sq}_{\#}(x) \mathrm{Sq}_{\#}(y), \quad 2<i<p .
$$

The proof is finished by separating each of the above equations into its homogeneous parts. 


\section{REFERENCES}

1. D. W. Anderson, A generalization of the Eilenberg-Moore spectral sequence, Bull. Amer. Math. Soc. 78 (1972), 784.

2. A. K. Bousfield and D. M. Kan, Homotopy limits, completions and localizations, Lecture Notes in Math., vol. 304, Springer-Verlag, Berlin and New York, 1972.

3. A. Dold, Über die Steenrodschen Kohomologieoperationen, Ann. of Math. (2) 73 (1961), 258-294.

4. W. G. Dwyer, Homotopy operations for simplicial commutative algebras, Trans. Amer. Math. Soc. 260 (1980), $421-435$.

5. S. Mac Lane, Homology, Springer-Verlag, New York, 1967.

6. J. P. May, Simplicial objects in algebraic topology, Van Nostrand, New York, 1967.

7. Applications, Lecture Notes in Math., vol. 168, Springer-Verlag, Berlin and New York, 1970, pp. 153-231.

8. D. Rector, Steenrod operations in the Eilenberg-Moore spectral sequence, Comment. Math. Helv. 45 (1970), 540-552.

9. W. Singer, Steenrod squares in spectral sequences. I, Trans. Amer. Math. Soc. 175 (1973), 327-336.

10. __ Steenrod squares in spectral sequences. II, Trans. Amer. Math. Soc. 175 (1973), 337-353.

11. L. Smith, On the Kunneth theorem. I, Math. Z. 116 (1970), 94-140.

Department of Mathematics, Yale Universtty, New Haven, Connecticut 06520 